\title{
Cascaded Solid-Spaced Filters for DWDM applications
}

\author{
Johan Floriot*, Fabien Lemarchand and Michel Lequime \\ Institut FRESNEL, UMR-CNRS 6133, ENSPM \\ D.U. de St Jerome, 13397 Marseille Cedex 20, France
}

\begin{abstract}
We report in this manuscript the study of solid-spaced Fabry-Perot filters. The use of high quality wafers as thick spacers and broadband dielectric mirrors with only few layers provides filters which have almost the same specifications as classical WDM interference filters. Multiple cavity filters, composed of single cavities of equal or different thick spacers are easy to manufacture and exhibit very low absorption and scattering losses. Experimental results concerning simple and double cavity filters with thick spacers centered at $1.56 \mu \mathrm{m}$ with a maximum transmission more than $98 \%$ and a full-width at half-maximum (FWHM) of about $0.5 \mu \mathrm{m}$ are exposed. We then propose different solutions for the extension to triple cavity filters with improved spectral properties.
\end{abstract}

Keywords : Thin-Film Filters, Solid-spaced etalons, DWDM

\section{INTRODUCTION}

The use of Wavelength Division Multiplexing (WDM) technique has provided with a strong improvement of the capacity of telecommunication networks since the beginning of the ' 90 s. This led to a reduction of spacing between adjacent communication channels and thus required the development of efficient filtering devices with flat and rectangular profiles, small bandwidths and high rejection ratios.

These narrow band-pass filters can be achieved using, among others, optical thin-film technology; but, a high number of layers (typically more than 100) are required to achieve the desired performance, which leads to large manufacturing time and increases the risk of errors during the deposition process. Moreover, the high intensity of the electric field inside the spacers magnifies the scattering losses induced by the interface roughness or volume inhomogeneities [1].

To circumvent these problems, we propose to use cascaded solid-spaced Fabry-Perot filters, each cavity including a high quality thin wafer (thickness between 50 and 150 microns) coated by dielectric mirrors with few layers (less than 10). We will demonstrate in this manuscript that we can fulfil the requirements of a 50-GHz DWDM filter with this kind of solution and give experimental results obtained on single and double cavity solid-spaced filters. Possible improvements of this basic configuration will also be discussed in the fourth part: the use of a blocking filter, wafers with different thicknesses or different mirrors with the same reflectance.

*johan.floriot@fresnel.fr 


\section{THEORETICAL APPROACH: DOUBLE SOLID SPACED FILTERS}

In thin-film technology, bandpass filters are usually composed of Fabry-Perot filters: one or several cavities (or spacers) are sandwiched between two reflectors [2]. Generally, the reflectors are dielectric mirrors made of a succession of layers, alternatively of high $(\mathrm{H})$ and low (L) index of refraction. Each layer is quarter-wave one, i.e. its optical thickness is $\lambda_{0} / 4$ where $\lambda_{0}$ is the deposition control wavelength. The spacer has an optical thickness of p $\lambda_{0} / 2$ (half-wave layer) where $\mathrm{p}$ is an integer. All these layers are deposited on a substrate (generally made of glass or fused silica). These filters are called all-deposited bandpass filters $[3,4]$.

In order to achieve the performances required for $50 \mathrm{GHz}$ WDM applications, the number of coated layers is high, typically more than 100. Indeed, to reach the required rejection ratio (typically, the intensity crosstalk of neighbouring channels must be at least $-20 /-25 \mathrm{~dB}$ bellow the target channel, even less for the following...), the bandwidth (typically >0.22nm@-0.5dB , <0.3nm@-3dB, <0.6nm@-20dB) and the steepness (bandwidth $<0.3 \mathrm{~nm} @$ $-3 \mathrm{~dB},<0.6 \mathrm{~nm} @-20 \mathrm{~dB})$, the number of cavities is at least equal to 3 with more than ten layers mirrors. Thus, the manufacturing of filters is time consuming and errors are likely to occur during the deposition process. Moreover, losses due to scattering, particularly in the spacer layer where the electric field is intense, may cause a severe fall of the maximum intensity for the target channel. Our solution is to use a high optical quality wafer (whose thickness is in the $100 \mu \mathrm{m}$ range) that will play the role of a thick spacer. Both sides of the wafer are polished, the parallelism between front and rear faces is about few arc-seconds, the absorption is null in the transmittance window. Then, dielectric mirrors are coated on this wafer. For our thick spacers, we choose to use fused silica, a classical optical material whose manufacturing and polishing processes are well controlled. These filters are called solid-spaced filters $(\mathrm{SSF})[3,4]$.

We consider now two wafers whose optical thicknesses are respectively $402 \lambda_{0} / 4$ (spacer1) and $540 \lambda_{0} / 4$ (spacer2) where $\lambda_{0}=1560.86 \mathrm{~nm}$ stands for the transmitted wavelength; the refraction index is supposed to be 1.44 . These specific retained values are those of the experimental demonstration of part III.

We will show that only 5 layers mirrors are required to reach the Full Width at Half Maximum (FWHM) of a WDM filter $(0.4 \mathrm{~nm})$. Indeed, let us consider the following double cavity filter: air / Filter1 / air / Filter2 / air where Filter 1 stands for HLHLH / spacer1 / HLHLH and Filter2 stands for HLHLH / spacer2 / HLHLH. The dielectric mirrors are composed of 5 layers (broadband mirrors); the materials corresponding to low and high refractive index are silica $\left(\mathrm{SiO}_{2}\right)$ and tantalum pentoxyde $\left(\mathrm{Ta}_{2} \mathrm{O}_{5}\right)$ respectively. The refractive index are respectively 1.46 and 2.09 at $1.56 \mu \mathrm{m}$.

The residual air gap between both coated wafers can be interpreted as the coupling layer of a two cavity filter. Consequently, its optical thickness should be quarter-wavelength.

We show on Fig.1 (black line) the spectral transmissions of the double cavity filter described above on a linear scale (zoom around the centered wavelength) and a dB scale over the whole ITU C grid.

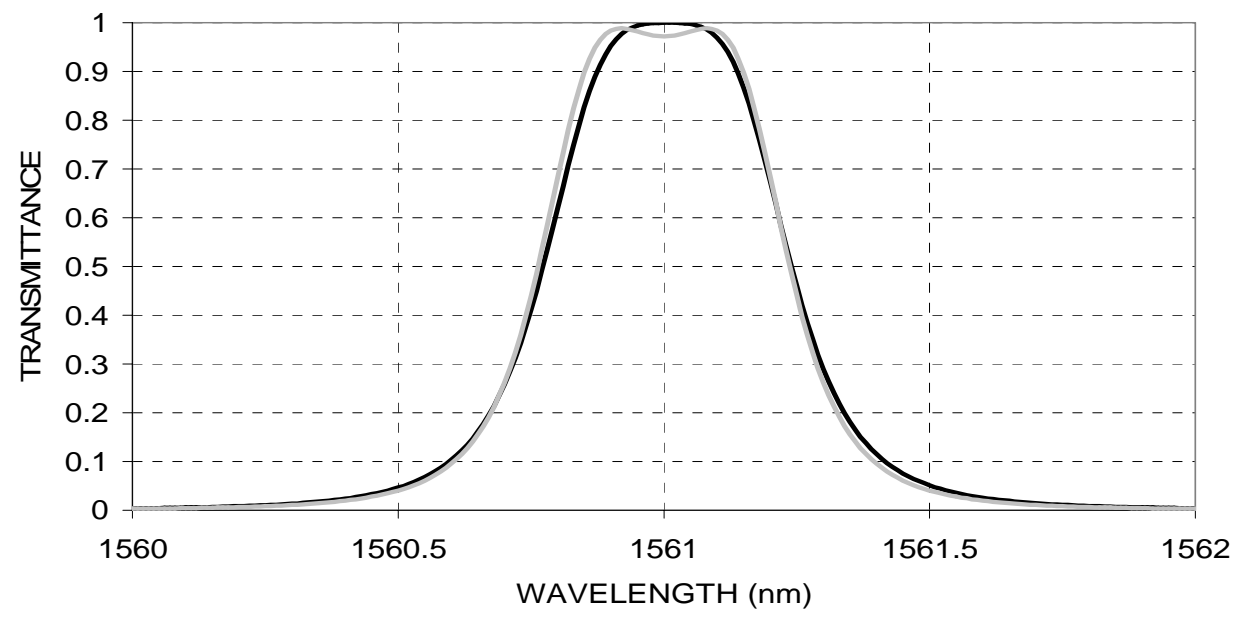




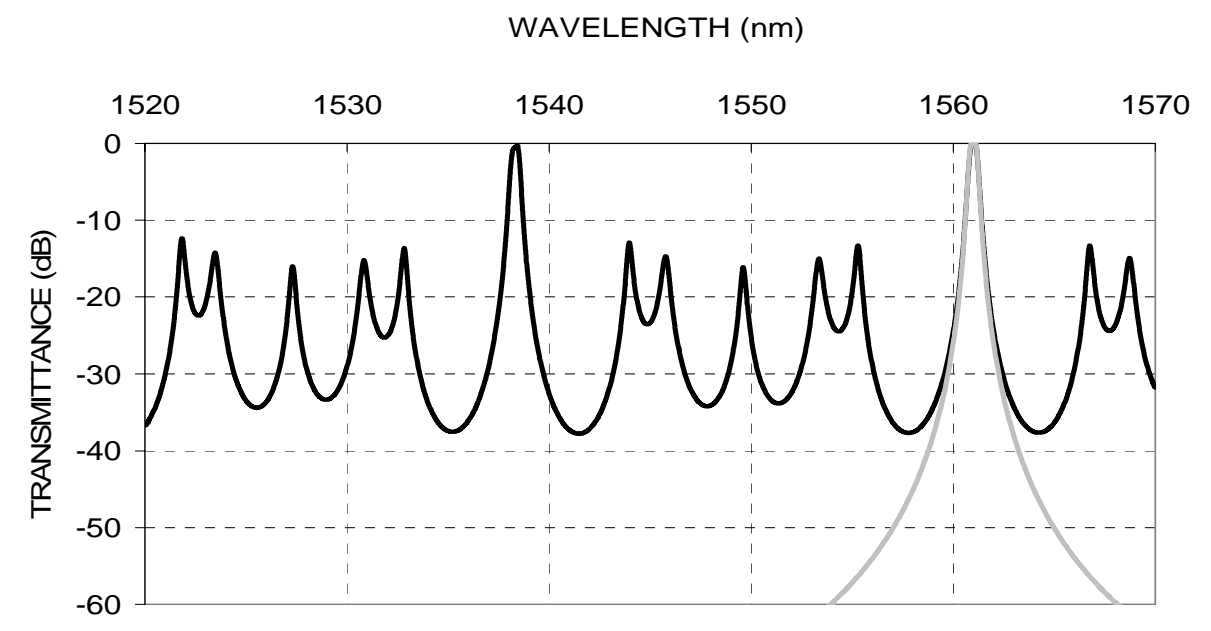

Fig.1: Theoretical transmission spectra for a double cavity solid-spaced filter (black line) and an all-deposited filter (grey line) on a linear and a dB scale

We can note that choosing different optical thicknesses for the spacers permits to introduce auto-filtering properties. The spectral transmissions of each filter coincide at a unique wavelength. The five neighbouring channels are strongly attenuated. The central-wavelength of the filter, roughly imposed by the optical thicknesses of the spacers is equal to $1560.86 \mathrm{~nm}$. The theoretical transmittance reaches $100 \%$ for the central-wavelength. The FWHM is $0.46 \mathrm{~nm}$. The residual transmission loss (RTL) is between $-12 \mathrm{~dB}$ (for the adjacent channel) and $-37 \mathrm{~dB}$ (directly at the base of the peak) for the whole C-band (1520-1570 nm) except near $1538.4 \mathrm{~nm}$ where a transmission band exists; at this particular wavelength, the RTL is only $-0.3 \mathrm{~dB}$ because the spectral transmissions of both spacers coincide once again.

As a comparison, we have shown on the same figure (grey line) the spectral transmission of an all-deposited double cavity filter with a similar FWHM. The materials are the same as above and the substrate is supposed to be made of glass (refractive index of 1.52). The design of the filter is: air/(HL) ${ }^{7} \mathrm{H} / 14 \mathrm{~L} / \mathrm{H}(\mathrm{LH})^{7} / \mathrm{L} /(\mathrm{HL})^{7} \mathrm{H} / 14 \mathrm{~L} / \mathrm{H}(\mathrm{LH})^{7} /$ substrate. The number of deposited layers is 63 and the total mechanical thickness is $21.2 \mu \mathrm{m}$, which is substantially more than the filter with thick spacers ( 20 deposited layers on each side of both thick spacers, which corresponds to a total mechanical deposited thickness of $4.4 \mu \mathrm{m}$ ). It is evident that the manufacturing time of the all-deposited filter is much more important (approximately 5 times higher). But, the graphs show clearly that the theoretical rejection outside the principal transmission band is better for the all-deposited filter (less than $-60 \mathrm{~dB}$ ).

Increasing the rejection of the solid-spaced filter is possible by introducing a blocking filter or adding a new cavity. This approach will be discussed in part 4. Consequently, it is clear that the manufacture of this filter should be more complex, expensive and time consuming.

\section{EXPERIMENTAL DEMONSTRATION OF SOLID SPACED FILTERS}

In a first step, the characterization of the wafers has to be made; their optical thickness at the center wavelength and at normal incidence, parameter of primary importance, is determined from transmittance measurements where interference effects occur resulting of the reflection between front and rear sides of the wafers. For that, we use a tunable laser source (EXFO FLS-2600) emitting in the C-band (1520-1570 nm). The wavelength repeatability is $10 \mathrm{pm}$. The laser beam is directed toward the wafer with a monomode fiber ended by a Lightpath ${ }^{\mathrm{TM}}$ pigtailed collimator. The beam waist, about $250 \mu \mathrm{m}$, is located on the wafer surface. Then, the transmitted light is collected with an identical collimator and a monomode fiber connected to an InGaAs photodiode followed by a low noise current amplifier and a 16 bit digital to analog converter. These working conditions are similar to those met in commercial WDM systems.

With the use of a least squares method all over the ITU C-band, we are able to determine the refractive index and the thickness of the wafers. 
Our measurements on the two commercial fused silica wafers that we have selected (nominal thicknesses of 107 and $145 \mu \mathrm{m}$ ) give a refractive index of 1.443 (we neglected dispersion effects) and thicknesses of $108.7 \mu \mathrm{m}$ and $146.0 \mu \mathrm{m}$. They also show that the specific wavelength 1560.86 is exactly half wavelength for the two wafers. This will be our target wavelength for the double coherent SSF. With the same technique, we are able to measure the thickness distribution of the wafers. We measure parallelism of about 3 arc-second.

The second step consists in the deposition of the dielectric mirrors on both sides of each wafer. The mirrors are five quarter-wavelength layer ones and are centered at $1561 \mathrm{~nm}$. The materials are $\mathrm{SiO}_{2}$ and $\mathrm{Ta}_{2} \mathrm{O}_{5}$ and the deposition process is IAD (Electron beam evaporation with Ion Assisted Deposition) with an in-situ optical monitoring. The characterizations of the filters are made with the same technique as described above. The figure 2 shows the transmission curves of both filters.
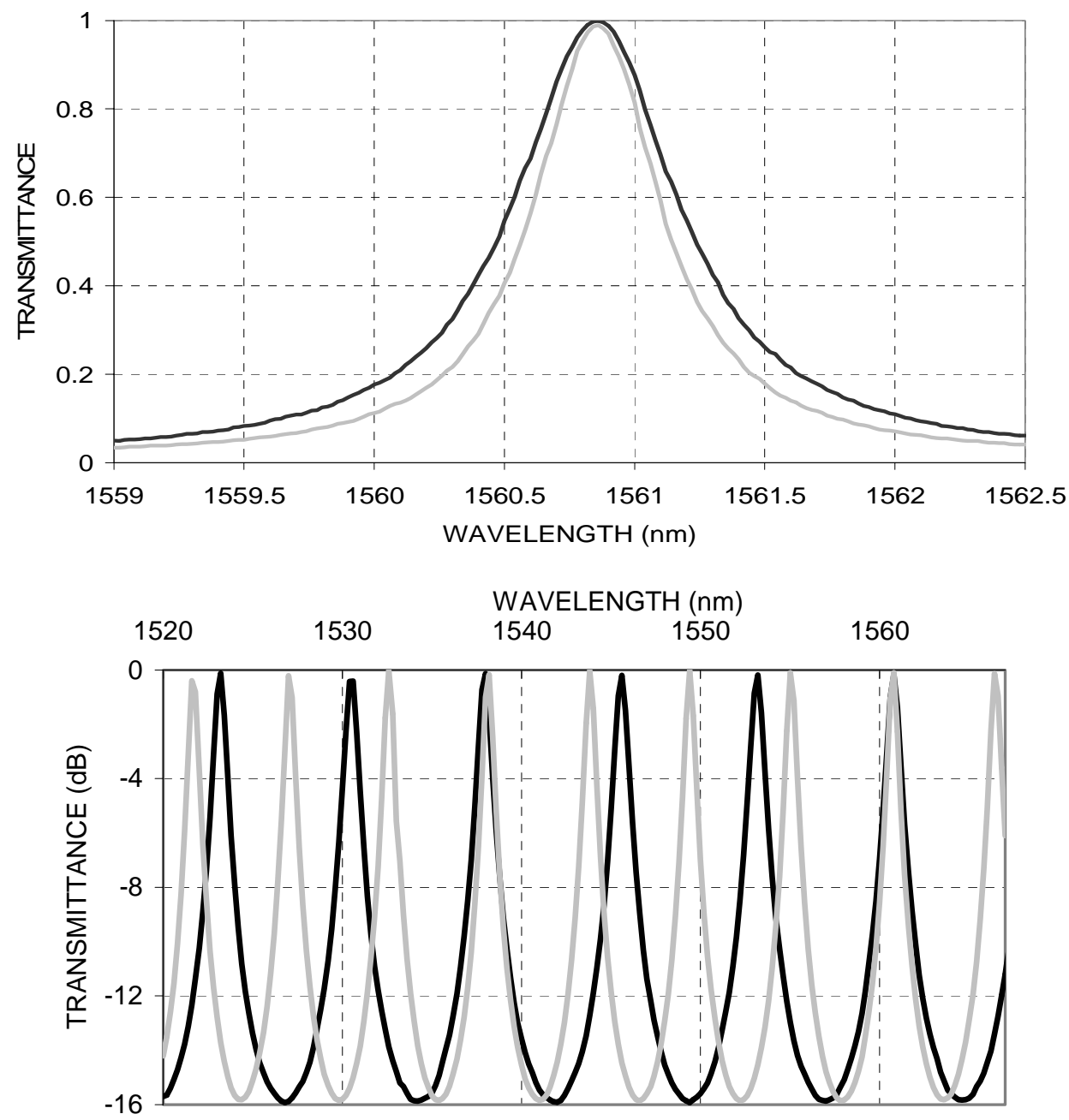

Fig.2: Experimental transmission spectra filter 1 (black line) and filter 2 (grey line) on a linear and a dB scale.

We can note first that the maximum transmission at $1560.86 \mathrm{~nm}$ is very close to unity, i.e. $99.9 \%$ for filter 1 (108.7 $\mu \mathrm{m}$ thick, black line) and $98.9 \%$ for filter $2(146.0 \mu \mathrm{m}$ thick, grey line). The FWHM are respectively 0.79 and $0.55 \mathrm{~nm}$, which is small considering that we use broadband mirrors. The rejection level is $-16 \mathrm{~dB}$. The maximum transmission is 
not $100 \%$ because of the small non-parallelism of the wafers and the finite size of the incident light (Gaussian distribution with a waist of $250 \mu \mathrm{m}$ ).

The coherent coupling of the two cavities is achieved thanks to a piezoelectric translator. We tune the air gap (about $30 \mu \mathrm{m})$ to a quarterwavelength optical thickness by optimizing the maximum transmittance for the central wavelength. The double cavity solid-spaced filter then obtained has the following transmission spectrum (figure 3).
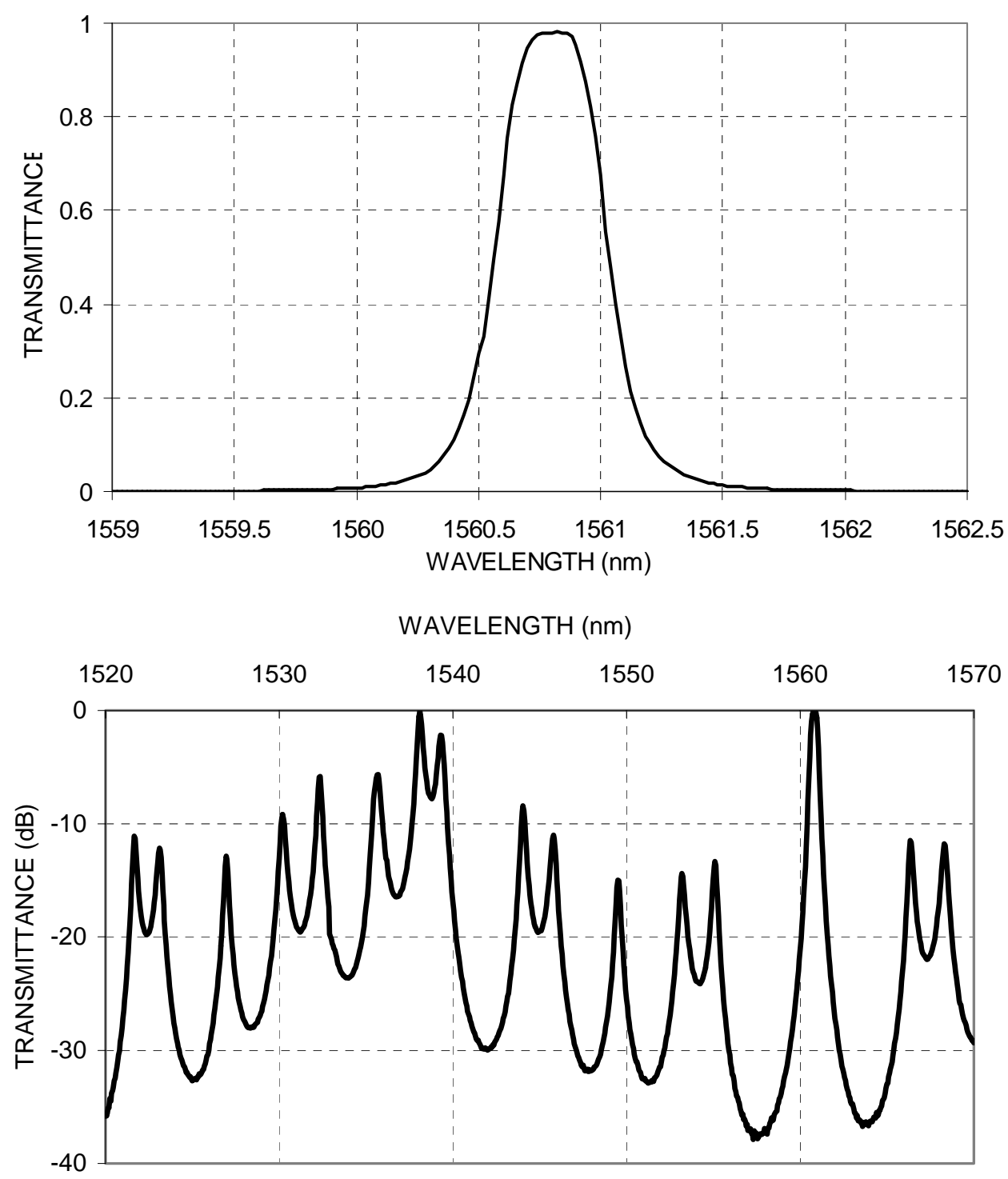

Fig.3: Experimental transmission spectrum for the double cavity filter

The center wavelength is still the same but the maximum transmission is reduced to $98.1 \%$ for the same reasons as above. The FWHM is $0.47 \mathrm{~nm}$, which is close to the theoretical prediction of $0.45 \mathrm{~nm}$. The steepness is increased and the profile is more rectangular. The rejection levels are $-14 \mathrm{~dB}$ for the adjacent channel $(1553.3 \mathrm{~nm}),-9$ $\mathrm{dB}$ at $1544.1 \mathrm{~nm}$ and only $-0.3 \mathrm{~dB}$ at $1538.2 \mathrm{~nm}$, where another coincidence exists. 
We can note that the agreement with the theoretical calculation (see part II, figure 1) is very good for the domain $1545-1570 \mathrm{~nm}$. Below $1545 \mathrm{~nm}$, differences appear that can be explained by introducing relative random errors in the range of $0-5 \%$ on the mirror layer thicknesses.

The transmission of such a filter shifts when the temperature varies. This property is of great importance for DWDM filters whose sensitivity to temperature must be less than $1 \mathrm{pm} /{ }^{\circ} \mathrm{C}$. Our filter has a sensitivity of about 11 $\mathrm{pm} /{ }^{\circ} \mathrm{C}$ (we have taken into account only the thermo-optic coefficient in a first approximation, knowing that its influence is dominant). This property could provide a method to tune the filter.

\section{TRIPLE CAVITY FILTERS WITH HIGH REJECTION BAND}

We now study different methods for increasing the rejection band and decreasing the intensity crosstalk. We have seen in part III that this point is one of the major drawbacks of our experimental demonstration.

First, let us consider the case of a typical triple cavity SSF whose cavities have the same thickness $(75.1 \mu \mathrm{m})$ and the mirrors are 7-layer ones. The materials are the same as mentioned above. We obtain the following results (Fig. 4):
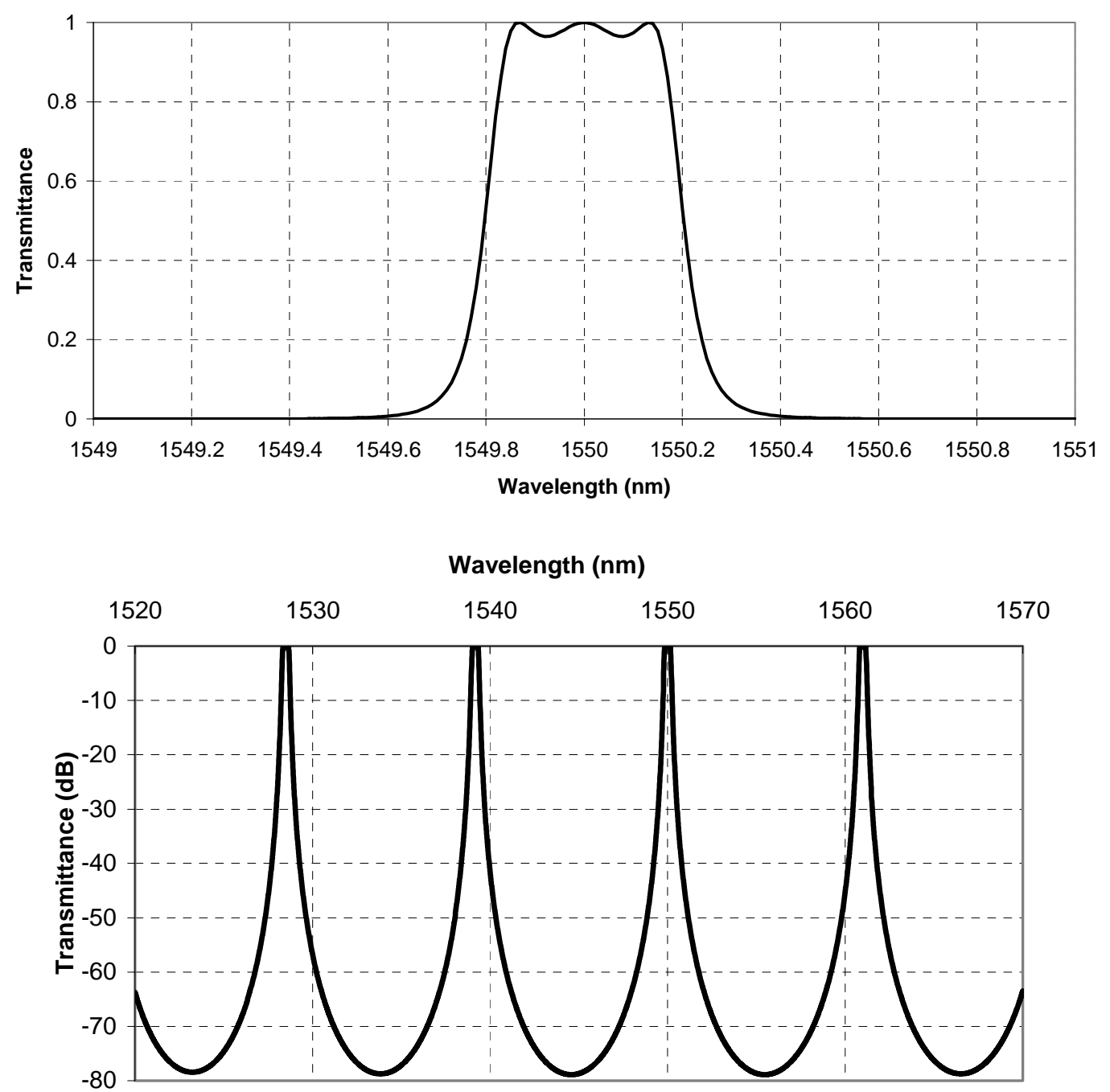

Fig.4: typical triple cavity filter with all cavities with the same optical thickness 
The maximum transmission is $100 \%$. The filter has some ripple in the transmission band. The rejection of this filter is $80 \mathrm{~dB}$ but all the secondary transmission bands are present. The FWHM is $0.4 \mathrm{~nm}$.

The first obvious solution for eliminating the secondary transmission bands consist in adding a blocking filter in front of our SSF. For example, we have considered a 11-layer all-deposited $\mathrm{Si} / \mathrm{SiO}_{2}$ filter (see [5] for more details). The use of $\mathrm{Si}$ (very high refractive index material) instead of $\mathrm{Ta}_{2} \mathrm{O}_{5}$ permits one to have a smaller number of layers. The spectrum of the triple cavity filter with the blocking filter is shown on figure 5 on a dB scale.

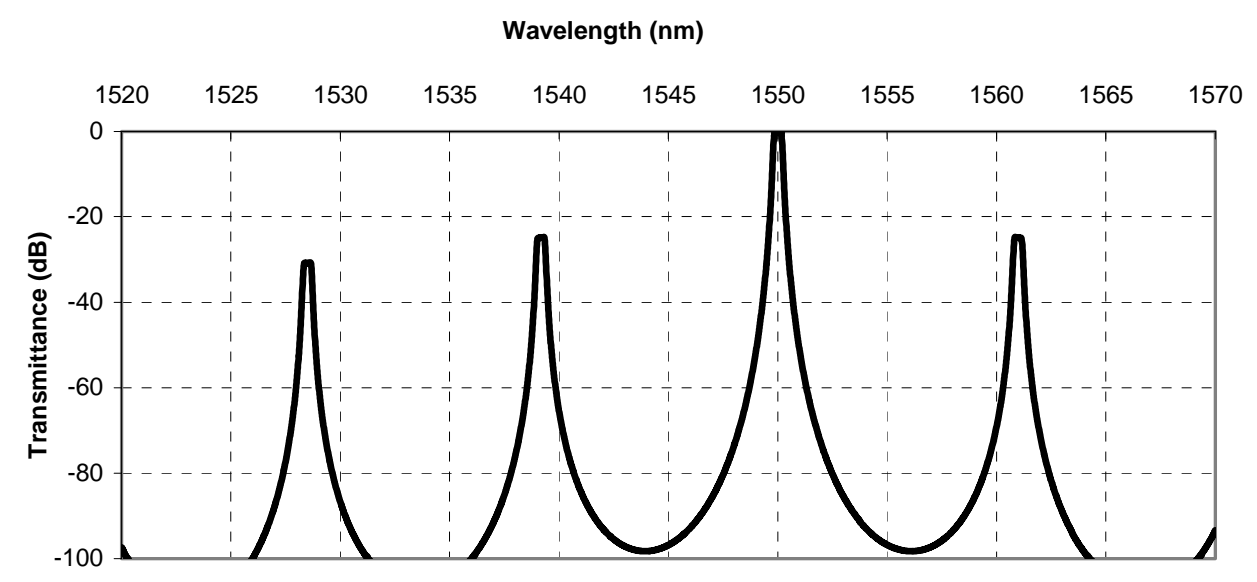

Fig.5: triple cavity filter with a $\mathrm{Si} / \mathrm{SiO}_{2}$ blocking filter

We remark that the rejection is strongly increased (typically less than $-30 \mathrm{~dB}$ for the whole C-band). The properties of the transmission band (FWHM, maximum transmission, steepness...) remain unchanged. The experimental study of such $\mathrm{Si} / \mathrm{SiO}_{2}$ filters is currently done in our laboratory.

Another solution to eliminate the non-desired transmission bands is to utilize cavities with different free spectral range, i.e. different thicknesses, exactly as we have done for the double cavity filter studied above. Each cavity is exactly half wavelength optical thickness for a unique wavelength in the ITU grid. Auto-filtering properties of the three cavities enable to improve the channel crosstalk.

One example still uses the same materials. The mirrors are 7 quarter-wavelength layer ones for which the optical thickness of the external layers are adjustable to obtain an antireflection effect in the transmission band. The thicknesses of the spacers are respectively $69.8,49.9$ and $74.6 \mu \mathrm{m}$. The center wavelength is $1550 \mathrm{~nm}$. The transmission spectra are shown on the figure 6 .

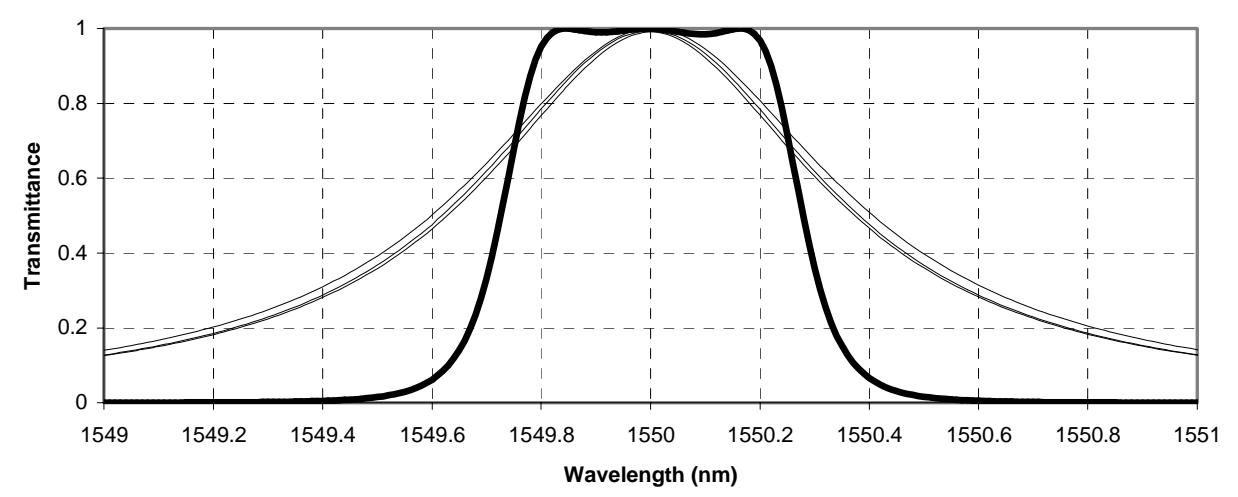




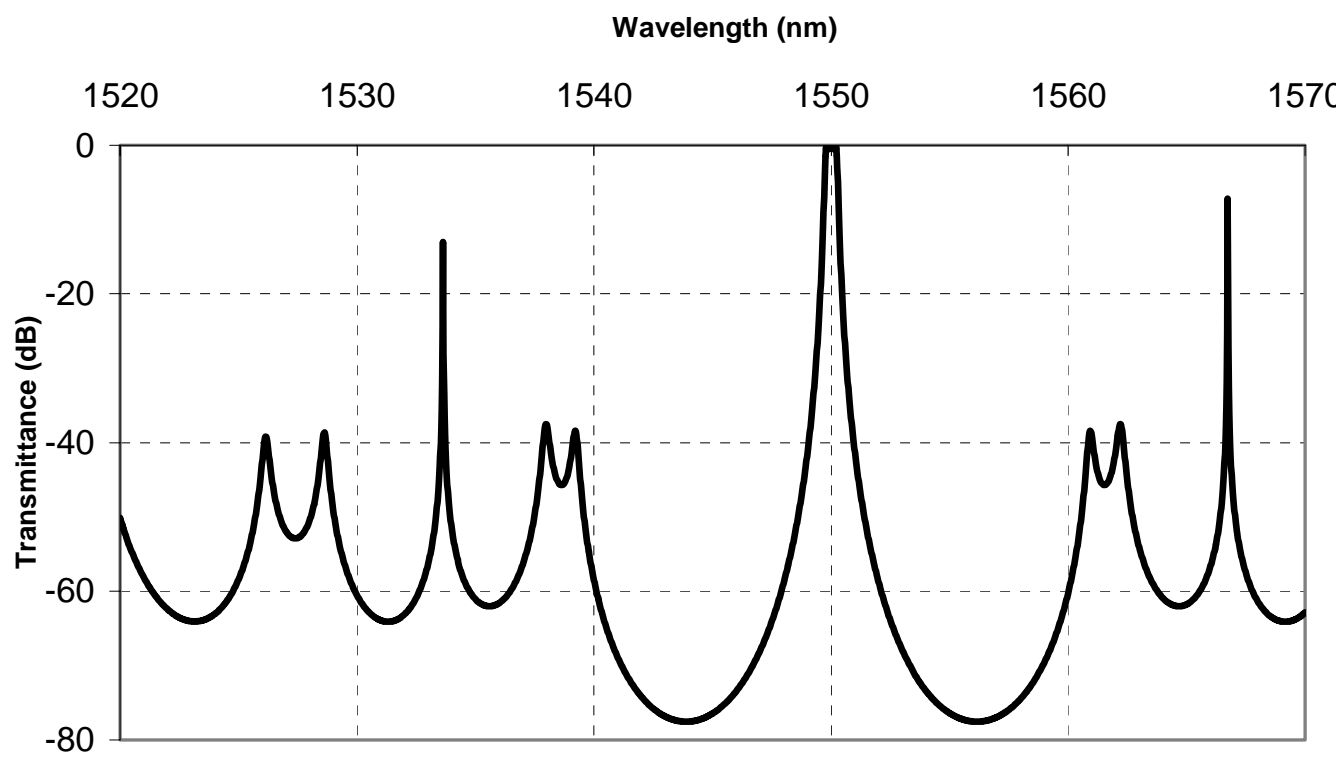

Fig.6: triple cavity solid-spaced filter

For clarity, we have plotted the spectra of the three simple cavity filters on the linear scale graph only (thin lines). The transmission is $100 \%$ for the center wavelength; the FWHM are respectively $0.72 \mathrm{~nm}$ for filter $1(69.8 \mu \mathrm{m}$ thick spacer) and 3 (74.6 $\mu \mathrm{m}$-thick spacer), $0.77 \mathrm{~nm}$ for filter $2(49.9 \mu \mathrm{m}$-thick spacer) and $0.54 \mathrm{~nm}$ for the triple cavity (thick black line). The rejection is less than $-30 \mathrm{~dB}$ on the whole C-band except around 1534 and $1567 \mathrm{~nm}$. The number of deposited layers is 42 and the total physical thickness to deposit is around $9 \mu \mathrm{m}$.

For the experimental demonstration of such a filter, we dispose of three wafers whose measured thicknesses are $69.3,49.4$ and $74.1 \mu \mathrm{m}$ respectively. Then, we need to adjust the optical thicknesses of the wafers to the desired ones. For that, we coat the wafers with a thin layer of silica whose physical thickness is in the $500 \mathrm{~nm}$ range. To control such a layer, we use our tunable laser source and we measure in-situ the transmission spectrum during the deposition. We observe the shift of the spectrum towards the higher wavelengths and we stop the process when we reach the convenient center wavelength $(1550 \mathrm{~nm})$. The difficulty is to attain the required precision which is about 2-3 nm. Experimental studies are in progress.

The last solution treated in this paper is the use of identical thick spacers, with different mirrors. More precisely, we consider dielectric mirrors with the same reflectance intensity but different phase dependence with respect to the wavelength. At the particular central wavelength, the phase of the complex reflection amplitude coefficient is equal to $0[\pi]$ (see for example [6]). But, if we design different dielectric mirrors, this phase can differ for the other transmissions bands. Indeed, the free spectral range is modified, and the resonant wavelengths do not coincide expect for the desired central wavelength.

As an illustration, let us consider a classical $(\mathrm{HL})^{\mathrm{m}}$ dielectric mirror and a $(\mathrm{HL})^{\mathrm{m}+\mathrm{q}} / 2 \mathrm{n} \mathrm{H} /(\mathrm{LH})^{\mathrm{q}}$ structure, where $\mathrm{q}, \mathrm{m}$ and $\mathrm{n}$ are integers. These designs present the same reflection coefficient at the central wavelength, but not the same phase dependence with wavelength $\mathrm{d} \varphi / \mathrm{d} \lambda$. For the second structure, the phase of the amplitude reflection coefficient varies more rapidly with wavelength around the center wavelength. The other wavelengths of maximum transmittance (given by eq 5 in [6]) are shifted. By cascading filters using such mirrors and combining different values of $\mathrm{n}$ and $\mathrm{q}$, we obtain an auto-filtering effect, even with same spacer thicknesses. We show in figure 7 the transmittance of a triple cascaded SSF with the same spacer thickness $(70 \mu \mathrm{m})$, and different mirrors as described above with $\mathrm{m}=7$. 


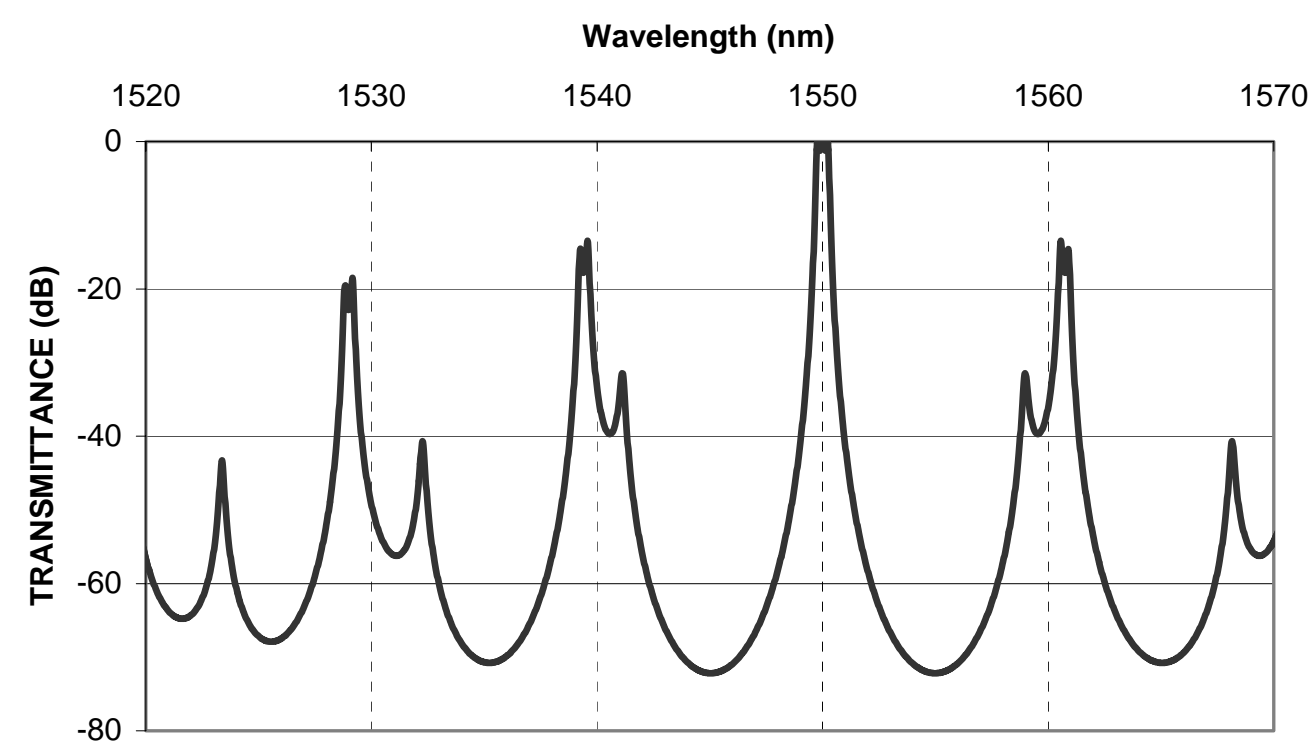

fig. 7 : triple cavities SSF with same spacer and different kinds of mirrors

The analysis of the theoretical spectral response shows that the residual transmission loss level is about $-20 \mathrm{~dB}$ for the ITU-C grid. With such designs, the rejection band is wide (1480nm -1620nm for our example) even with identical thick spacers. An experimental illustration of such designs is currently in progress.

\section{CONCLUSION}

We have demonstrated the feasibility of a double cavity solid-spaced filter. We have exposed the experimental results we obtained for simple cavity filters and double cavity filter. In the fourth part, we have optimized the design of a triple cavity filter in order to increase the spectral properties of the precedent filter (mainly, channel crosstalk). Three solutions are given. The experimental realization of these filters is in progress in our laboratory.

Compactness is of prime importance for WDM components because of the miniaturization constraints of commercial networks. Thus, we investigate the possibility to adhere such simple cavity filters by optical contacting techniques.

\section{REFERENCES}

[1] M. Lequime, C. Deumié, C. Amra Proceedings of SPIE 3738, 268-277 (1999)

[2] H.A. Macleod, Thin-Film Optical Filter (Macmillan), $3^{\text {rd }}$ ed., Institute of Physics Pub., New York, 2001, pp. 12-66, 179-185, 257-345

[3] M. Bass, E.W. Van Sryland, Handbook of Optics, Vol. 1, $2^{\text {nd }}$ ed., 42-12, McGraw-Hill Professionnal, 1994

[4] A. Roche, A. Title, Appl. Opt. 14 (1975) 765

[5] J. Floriot, F. Lemarchand, M. Lequime, Opt. Comm. 222 (2003) 101-106

[6] M. Lequime, R. Parmentier, F. Lemarchand, C. Amra, Appl. Opt. 41 (2002) 3277 